\title{
Institucionalização de Processos no Setor de Produção de Software de uma Universidade Federal
}

\author{
Fabiana Freitas Mendes ${ }^{2}$, Patrícia Gomes Fernandes ${ }^{3}$, Caroline da Cunha Mota ${ }^{1}$, \\ Rosângela da Silva Nunes ${ }^{1}$, Maria Dalva S. Martins ${ }^{1}$. \\ ${ }^{1}$ Centro de Recursos Computacionais (CERCOMP)- Universidade Federal de Goiás \\ (UFG) - Caixa Postal 131 - 74.001-970 - Goiânia- GO - Brasil \\ ${ }^{2}$ Instituto de Computação (IC) - Universidade Federal do Mato Grosso (UFMT) \\ Av. Fernando Corrêa da Costa, 2367. B. Boa Esperança - 78060-900- Cuiabá- MT- \\ Brasil \\ ${ }^{3}$ Faculdade de Computação (Facom) - Universidade Federal de Mato Grosso do Sul \\ (UFMS) - Caixa Postal 549 - 79.070-900 - Campo Grande - MS - Brasil \\ fabianadic.ufmt.br, patriciadfacom.ufms.br, \\ \{carolinecmota,rosa.cercomp, dalvinha\} egmail.com
}

\begin{abstract}
In order to be successful, a software process improvement initiative includes the institutionalization of processes that are composed of software engineering good practices. This study presents the difficulties found in the software processes' institutionalization that took place at the Centro de Recursos Computacionais, Universidade Federal de Goiás, as well as the actions executed to overcome these difficulties. It was necessary to change processes and also the organizational structure and the distribution of the systems' responsabilities among employees.
\end{abstract}

Resumo. A fim de ser bem sucedida, uma iniciativa de melhoria de processos de software envolve a institucionalização de processos que incorporem boas práticas de engenharia de software. O presente trabalho apresenta as dificuldades relacionadas à institucionalização do processo de software do Centro de Recursos Computacionais da Universidade Federal de Goiás, bem como as ações que estão sendo executadas para tratá-las. Mais do que lidar com os processos, foi necessário lidar com a estrutura organizacional e com a distribuição de responsabilidades sobre os sistemas entre os seus colaboradores.

\section{Introdução}

Segundo [ISO/IEC 2008] processo é um conjunto de tarefas inter-relacionadas que transformam entradas em saídas. Em empresas de software, estes processos transformam as ideias ou requisições (entradas) dos clientes em código objeto. Muitos são os processos relacionados à construção de software, como o processo de análise de requisitos, design da arquitetura de software e gerência de projeto de software.

Baseados na hipótese de que a qualidade do processo de software influencia a qualidade dos produtos [TYRRELL 2000], muitas organizações têm investido esforços em Melhoria de Processos de Software (MPS). Um projeto de MPS tem como objetivo conhecer, definir e/ou melhorar os processos relacionados ao desenvolvimento de software para torná-los mais efetivos e eficientes. 
As Instituições Federais de Ensino Superior (IFES) têm crescido significantemente e também a demanda por sistemas corporativos informatizados que apoiem os seus processos organizacionais. Várias IFES têm desenvolvido internamente sistemas informatizados para atender às suas demandas. Entretanto, a falta de recursos humanos e infraestrutura dificultam a sua continuidade.

Nesse contexto, existe a demanda por um projeto de MPS em instituições federais que, como a Universidade Federal de Goiás (UFG), possuem um setor de desenvolvimento de software. Na UFG o setor, denominado Centro de Recursos Computacionais (CERCOMP), é responsável pelo desenvolvimento e manutenção de 43 sistemas com uma equipe de 29 pessoas.

Desde o ano de 2007, o CERCOMP investe em MPS, tendo sido executados três ciclos de melhoria [MENDES et al 2010]. Contudo, o maior desafio atual do projeto é a institucionalização dos processos já definidos.

A institucionalização de um processo diz respeito a quanto ele está arraigado no modo com que o trabalho é executado em uma organização, que é o reflexo da sua execução com consistência e comprometimento [SEI 2006]. Em outras palavras, um processo está institucionalizado se é sistematicamente seguido, ainda que com o tempo mudem os detalhes de implementação [PAULK 2002].

Elementos que apoiam a institucionalização estão presentes em modelos de maturidade de processos de software utilizados na indústria. No CMMI, por exemplo, as metas genéricas incorporam o grau de institucionalização de um processo, permitindo caracterizar sua progressão [SEI 2006]. Já o MPS incorpora a institucionalização no conceito de capacidade de processo [SOFTEX 2009]. Da mesma forma que no CMMI, no MPS grau de institucionalização deve aumentar conforme são galgados os níveis de maturidade superiores. O refinamento dos diferentes níveis de capacidade é apresentado na forma de atributos de processo, que podem ser equiparados às metas genéricas do CMMI. A cada atributo de processo estão associados um ou mais resultados esperados de processo, que devem ser satisfeitos para o alcance do nível de capacidade desejado.

Este artigo tem como objetivo mostrar como tem sido tratada a necessidade de institucionalização dos processos do CERCOMP. Para tanto, a Seção 2 apresenta pesquisas realizadas para avaliar os problemas relacionados à institucionalização dos processos do CERCOMP. Já a Seção 3 apresenta a estratégia de institucionalização criada para lidar com os problemas detectados nas pesquisas. Na Seção 4 são apresentados alguns resultados e dificuldades enfrentadas com a execução da estratégia e a Seção 5 apresenta algumas considerações finais relacionadas ao trabalho aqui descrito.

\section{Pesquisa sobre Institucionalização}

No final do ano de 2009, apesar da iniciativa de dois anos de duração, os processos até então definidos ainda não eram utilizados por toda a equipe da Divisão de Sistemas [MENDES et al 2010a]. Neste momento já estavam sendo encerradas as análises de ferramentas para apoio ao processo [MENDES et al 2010b]. Todavia, apenas alguns projetos utilizavam o processo e, ainda assim, não o utilizavam completamente.

Por esse motivo, foi planejada e realizada uma pesquisa com o intuito de avaliar a percepção, coletar opiniões e identificar os problemas e dificuldades associados aos processos de software definidos para a Divisão de Sistemas do CERCOMP.

Para tanto, o Grupo de Processos de Software (GPS) convidou todos os 
colaboradores da Divisão de Sistemas, o diretor e o coordenador do Sistema de Atendimento ao Usuário $\left(\mathrm{SAU}^{1}\right)$ para participar da pesquisa. Apesar disso, nem todos participaram. No total, 25 funcionários foram consultados durante a pesquisa.

A pesquisa foi feita por meio de uma entrevista guiada por um questionário com dezoito perguntas que tinham como objetivo:

- Caracterizar o modo de trabalho atual, com o objetivo de analisar a distância com o modo de trabalho estabelecido no Manual de Produção de Software - que descreve o processo-padrão da organização;

- Identificar, dos processos presentes no Manual de Produção de Software, os mais conhecidos pela equipe, modo como o conhecimento foi adquirido e aqueles que são mais utilizados;

- Identificar o grau de satisfação da equipe com o processo definido, destacando os ponto fracos e oportunidades de melhoria.

Depois desta primeira consulta à equipe e da apresentação dos resultados a todos da Divisão de Sistemas, foi percebida a necessidade de informações adicionais sobre os sistemas em desenvolvimento e manutenção. Por isso, foi feita uma segunda pesquisa com o objetivo de conhecer melhor os sistemas da Divisão e o modo como a equipe estava distribuindo o trabalho de desenvolvimento ou manutenção entre sistemas.

Nesta segunda pesquisa, entretanto, foram entrevistados apenas os funcionários que possuíam a função de Gerente de Produto e, quando fossem necessárias informações que eles não pudessem fornecer, também foram consultados outros membros dos projetos de manutenção ou desenvolvimento do sistema em questão.

As pesquisas realizadas mostraram que o conhecimento dos sistemas encontravase concentrado e, por causa disso, havia dependência de pessoas específicas. Além disso, o tamanho da maioria das equipes de desenvolvimento ou manutenção dos sistemas era de apenas uma pessoa ("euquipes"), fato que dificultava ainda mais a aplicação do processo. A existência de euquipes demonstrava que o processo criado era inadequado, pois requer diversos papéis durante um ciclo de execução.

De acordo com Schein, para que uma mudança cultural ocorra, é necessário que os envolvidos se sintam desconfortáveis com a situação atual, por não atender aos objetivos organizacionais [Schein 2004]. Entretanto, os colaboradores se mostraram satisfeitos com a forma de trabalho atual. Dessa forma, era bem provável que os processos definidos não seriam adotados pelos colaboradores.

Os resultados das pesquisas realizadas no CERCOMP juntamente com o modelo dos questionários utilizados podem ser encontrados em [GPS 2010]. A próxima seção apresentará as ações criadas para lidar com os principais problemas detectados relacionados à institucionalização do processo no CERCOMP.

\section{Ações para Institucionalização do Processo}

Para lidar com os problemas relatados na seção anterior foi realizado um planejamento da reestruturação da Divisão de Sistemas, concentrando-se inicialmente nos papéis gerenciais e de analista. Os objetivos da reestruturação são: disseminar o conhecimento dos processos e dos sistemas; diminuir a dependência de pessoas específicas e o número

1 SAU recebe as requisições dos usuários e as repassam para a divisão responsável por atendê-las. 
de equipes com uma única pessoa ("euquipes”); melhorar e institucionalizar o processopadrão contido no Manual de Produção de Software.

Paralelamente à reestruturação, foi planejada a revisão do processo descrito no Manual, com o objetivo de simplificá-lo. Posterior à execução da estratégia aqui descrita, será desenvolvida um outra estratégia para lidar com os papéis de técnicos.

Atualmente a equipe da Divisão de Sistemas está organizada em relação aos seus sistemas. Para cada sistema, são alocadas pessoas encarregadas ou por desenvolvê-lo (no caso da não existência do produto) ou por mantê-lo. O número total de sistemas atual da Divisão é 43 , sendo que destes sistemas, $75 \%$ são mantidos por equipes com apenas uma pessoa. Além disso, a interação entre as pessoas responsáveis por cada sistema é pequena.

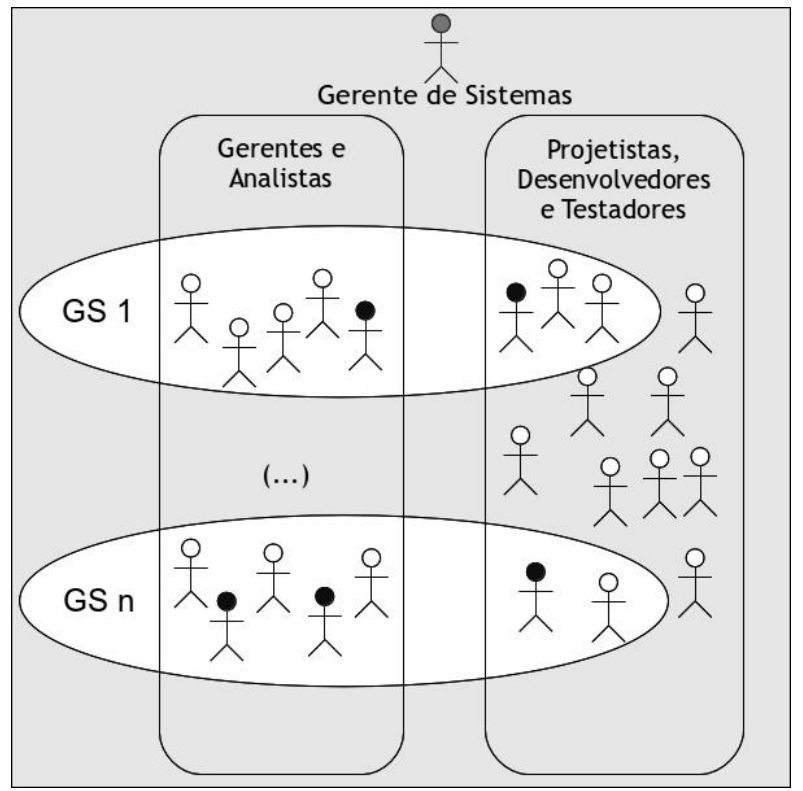

Figura 1: Cenário estrutural intermediário Legenda: $G S$ = Grupo de Similaridade

Os problemas relacionados à maneira como atualmente a Divisão de Sistemas está organizada são: concentração de conhecimento, sobrecarga de funções, existência de equipes com apenas uma pessoa e dificuldade de visualizar e gerenciar o trabalho desenvolvido por cada funcionário da Divisão. Para solucionar estes problemas, foi proposta uma nova estrutura organizacional, apresentada na Figura 1.

Como pode ser visto, será formado um grupo de analistas e gerentes, e um outro grupo com o restante da equipe. Assim, para manutenir ou desenvolver sistemas, serão necessárias pelo menos uma pessoa de cada um destes dois grupos. Depois da execução da proposta apresentada por este artigo, pretende-se executar um outro ciclo de reestruturação que lidará com o restante da equipe. No final, espera-se que exista pelo menos três grandes grupos de papéis: analistas e gerentes, projetistas, e desenvolvedores e testadores.

Para se chegar ao cenário apresentando pela Figura 1, foi criado um conjunto de passos denominados em conjunto de Estratégia de Reestruturação da Equipe, ilustrado pela Figura 2.

Como pode ser visto, o primeiro grande conjunto de passos é denominado 
Determinação Estratégica, que possui como ações:

1. Classificar pessoas por funções: identificar para cada um dos funcionários da Divisão, as funções que ele deseja executar. Existem três grandes grupos de funções: grupo de analistas e gerentes, grupo de projetistas e desenvolvedores, e grupo de testadores.

Figura 2: Fluxograma da Estratégia de Reestruturação

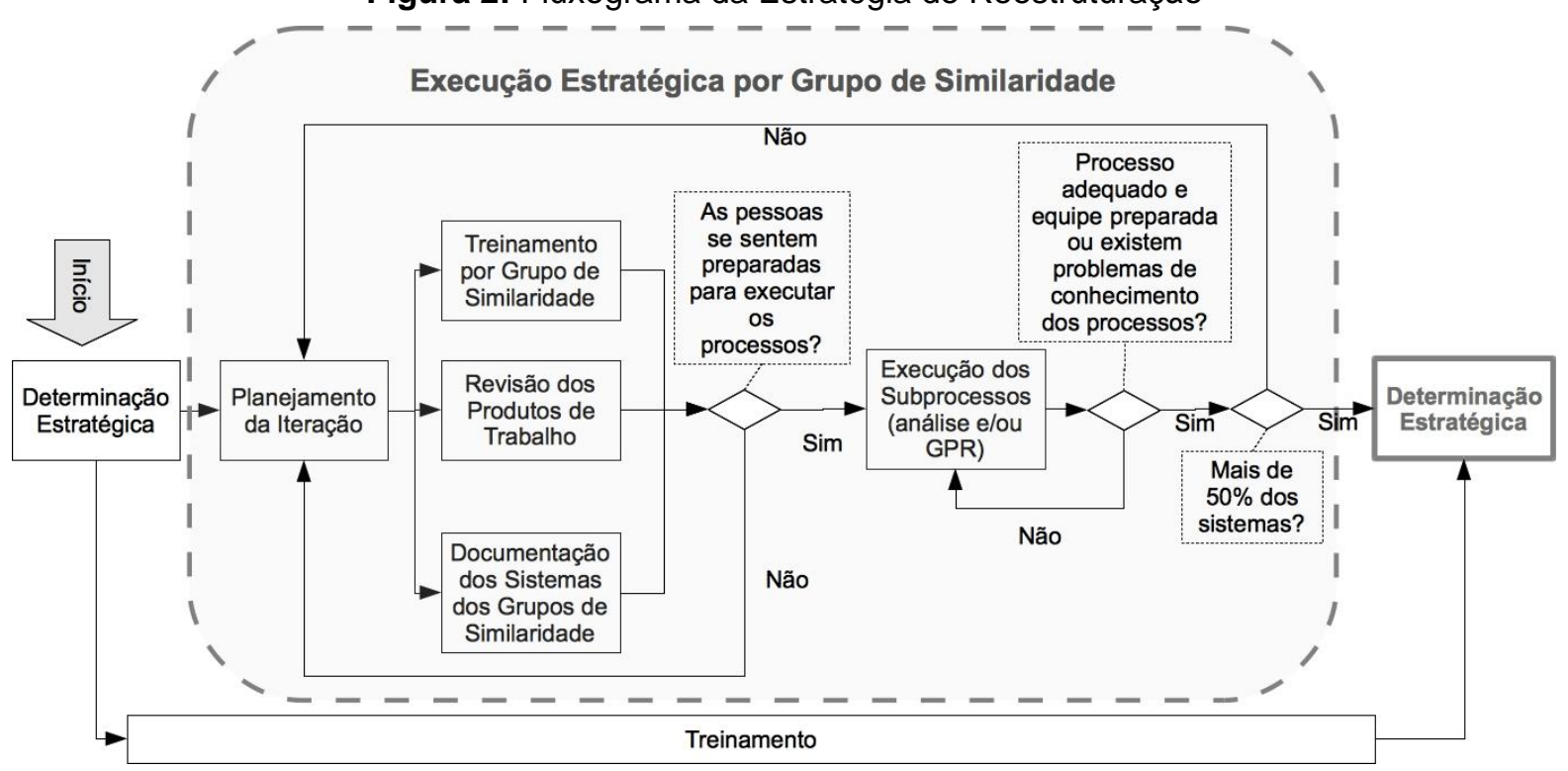

2. Classificar sistemas por similaridade: classificar os sistemas levando em consideração a similaridade em relação ao negócio com o qual o sistema lida (sistemas acadêmicos, administrativos, etc). Além disso, para montar os GS (Grupos de Similaridade) é considerada a quantidade de requisições e usuário do sistema.

3. Apresentar planejamento para Divisão de Sistemas: a apresentação deve ser realizada antes da execução dos dois passos posteriores à abordagem.

Após a Determinação Estratégica, tem início o Treinamento que deve ser executado durante todo o período de reestruturação. As principais ações deste passo são:

1. Definir tópicos de treinamento, levando em consideração as funções e as tecnologias adotadas pelo CERCOMP. Planejar treinamentos básicos, na forma de oficinas, com o intuito de nivelar o pessoal em relação a conceitos básicos associados a processo, qualidade, dentre outros. O objetivo adicional é promover nas pessoas motivação para desenvolver e manter software de boa qualidade.

2. Capacitar os funcionários de acordo com a função a ser desempenhada por eles. Executados pelo GPS ou contratados pelo CERCOMP, de acordo com o seu tipo.

Paralelo ao treinamento, serão executados ciclos com o objetivo de disseminar o conhecimento dos produtos pelos GS alvo e de auxiliar a execução do processo nestes mesmos grupos. Cada ciclo é composto por cinco passos:

1. Planejamento da iteração. Este passo é composto pelos seguintes sub-passos:

a) Definir quais documentos de análise deverão ser gerados para os sistemas. $O$ objetivo é documentar os produtos, de modo a facilitar a manutenção. Estes 
documentos, entretanto, só serão gerados caso exista, no GS em questão, uma pessoa extra responsável por realizar esta documentação.

b) Definir quantos e quais sistemas entrarão no GS que será o alvo da estruturação da iteração. A agregação dos sistemas aos GS e à nova estrutura é feita de forma gradual. A cada iteração, acrescenta-se mais sistemas a um GS. Por exemplo: considere um cenário de oito sistemas administrativos: inicialmente, seriam formados quatro GS, cada um contendo dois sistemas. Ao final de uma iteração, seriam formados dois GS com os oito sistemas administrativos. Finalmente, na terceira iteração, haveria apenas um GS com todos os sistemas administrativos. É importante a seleção de sistemas menos críticos e a contratação de pelo menos um funcionário para que não haja sobrecarga de trabalho.

c) Distribuir pessoas pelos GS criados. É importante que, neste momento, seja definido o Gerente dos Produtos do GS. Este papel ajudará a compartilhar o conhecimento relacionado aos produtos.

2. Treinamento por GS. Diferentemente da fase de treinamento apresentada anteriormente, o objetivo aqui é que cada funcionário de cada GS entenda todos os sistemas do GS a que pertence. É importante que exista nestes treinamentos uma pessoa do SAU e que toda documentação gerada no passo 4 seja passada para eles.

3. Revisão dos produtos de trabalho. Este passo consiste na revisão dos produtos de trabalho gerados durante o período de existência do GS. A lista de produtos de trabalho a serem revistos deve ser decidida durante o planejamento da iteração.

4. Documentação dos sistemas dos GS. Este passo é realizado desde que haja contratação de uma pessoa extra e em paralelo com os dois anteriores $\mathrm{O}$ objetivo é desenvolver documentação de requisitos para os sistemas dos GS.

Caso seja notado que os colaboradores do GS já conhecem os sistemas do grupo, então o passo cinco será executado. Caso contrário, será executada uma nova iteração composta dos passos 1 a 4 apresentados anteriormente.

5. Execução dos subprocessos (Análise e/ou Gerência de Projeto). Após a disseminação do conhecimento, devem ser executados os processos contidos no Manual de Produção de Software. Nos passos anteriores estes processos já poderiam ter sido executados, mas a ênfase é que, neste momento, sejam executados os processos de acordo com o que está contido no Manual. Neste passo, os processos de análise e gerência de projeto são executados no GS até que a equipe considere que o processo está adequado e se sinta preparada para executá-los.

Estes cinco passos devem ser executados até que 50\% dos sistemas do CERCOMP sejam afetados pela execução da estratégia. Quando este marco for atingido o GPS deverá pensar em uma estratégia para lidar com os funcionários que executam as funções de projetista, desenvolvedor e testador.

O Gerente de Produto, representado na Figura 1 por bonecos escuros dentro dos retângulos, tem a função de facilitar a disseminação do conhecimento dos sistemas.

\section{Resultados e Dificuldades}

A estratégia apresentada na seção se encontra em execução desde novembro de 2010. Atualmente estão sendo executados dois GS, contendo cinco dos sistemas do 
CERCOMP. Ambos grupos estão executando os ciclos descritos na estratégia.

O primeiro GS possui dois sistemas, e o outro três. No total, estão envolvidas nove pessoas, sendo que duas delas atuam como mediador (papel responsável por gerenciar as ações de reestruturação), quatro gerentes de produto e três analistas.

Para a documentação estão sendo utilizados documento visão, lista de itens de menu, regras de negócio e MER. Em relação a treinamentos, foram feitas apresentações formais ou não para que cada componente de cada GS conhecesse todos os sistemas. Além disso, para disseminação de conhecimento, está-se procurando resolver todos os problemas relacionados aos sistemas de um GS dentro do GS em questão.

Nestas instâncias a estratégia apresentada na seção anterior foi ligeiramente alterada, devido à não contratação de uma pessoa extra para cada GS, conforme planejado.

Apesar do pouco tempo de execução da estratégia, já é possível visualizar alguns resultados como um maior conhecimento da equipe em relação ao processo definido e a própria conscientização da necessidade de documentação. Além disso, problemas que se prolongavam há mais de um ano foram resolvidos através da interação promovida dentro de um dos GS. Lentamente, o conhecimento dos sistemas tem sido compartilhado entre a equipe e as pessoas tem interagido mais.

Entretanto, diversas foram as dificuldades enfrentadas durante o período de execução da Estratégia de Reestruturação. Estas dificuldades geraram algumas ações, descritas a seguir:

- Dificuldade de promover o trabalho em equipe. Por existirem muitas "euquipes", os funcionários estavam acostumados a trabalhar individualmente. Com os GS foi necessária a interação entre os membros das equipes de cada um dos sistemas do GS. Entretanto, a forma de trabalho antiga estava arraigada na equipe. Assim, foi necessário desenvolver estratégias como a instituição de reuniões periódicas, fazer com que pessoas chave do GS tivessem suas mesas de trabalho mais próximas, e criar a gerência do GS, que tem como um dos objetivos detectar os problemas de interação e promover ações para tratá-los.

- Falta de prioridade das atividades da Estratégia de Reestruturação. As atividades de reestruturação são deixadas de lado para atender demandas de outros projetos da instituição. A solução deste problema passa pelo convencimento das pessoas da emergente necessidade da reestruturação da equipe e do apoio da direção nestas ações. Espera-se que este apoio e convencimento seja feito à medida que mais pessoas forem compondo os grupos e os resultados forem alcançados.

- Falta de acompanhamento das atividades de Reestruturação. Para lidar com este problema, foi designada uma pessoa para acompanhar as atividades do GS. Este papel é responsável, dentre outros, por agendar reuniões, e elaborar e divulgar as atas de reunião.

\section{Considerações Finais}

Este trabalho mostrou como estão sendo organizados os esforços para a institucionalização dos processos do CERCOMP. Apesar da existência de um projeto de MPS com mais de três anos de duração, os processos definidos ainda não são amplamente utilizados na organização. Para lidar com este problema, foram realizadas 
duas pesquisas com o objetivo de melhor entender os motivos da não utilização do processo. Depois disso, foi elaborada uma estratégia que, como resultado principal, deve promover a utilização dos processos por toda a equipe.

Atualmente, a estratégia desenvolvida está sendo executada por dois grupos de similaridade. Os principais problemas até então enfrentados e as ações criadas para lidar com eles também foram apresentados neste trabalho. Entretanto, para a obtenção dos resultados pretendidos pela Estratégia de Reestruturação, ela precisa ser executada mais amplamente no CERCOMP.

\section{Referências}

GPS, Grupo de Processos de Software do Cercomp (2010). Pesquisa sobre a institucionalização do Processo. disponível em http://www.cercomp.ufg.br/?noticia=1298546258\&site_id=17. Acesso em março de 2011.

ISO/IEC, International Organization for Standardization/ International Electrotechnical Comission. (2008) ISO/IEC 12207 Systems and software engineering- Software life cycle processes, Geneve: ISO, 2008.

MENDES, F. F., NASCIMENTO, H. A. N. D., FERNANDES, P. G., NUNES, R. S., MOTA, C. C. (2010). Implantação de Melhoria de Processos em um Setor de Produção de Software de uma Universidade Federal. IX Simpósio Brasileiro de Qualidade de Software, p. 359-365.

MENDES, F. M.; FERNANDES, P. G.; OLIVEIRA, J. L.; MOTA C. C.; MARTINS M. D. S.; NUNES R. S (2010). Análise de Ferramentas para Apoio a Gerência de Projetos e Gerência de Requisitos de Software. VI Workshop Anual do MPS 2010, p. 146-155.

PAULK, M. C.(2002) What Does 'Institutionalization' Means? Software Quality, p. 13-14, 2002.

SCHEIN, E. (2004) Organizational Culture and Leadership. Jossey-Bass, 2004.

SEI - Software Engineering Institute (2006). CMMI for Development, versão 1.2. Technical Report CMU/SEI-2006-TR-008, Software Engineering Institute/Carnegie Mellon University, 2006.

SOFTEX, Associação Para Promoção da Excelência do Software Brasileiro (2009). Melhoria de Processo de Software Brasileiro (MPS.BR): Guia Geral:2009. Disponível em: <http://www.softex.br/mpsbr/guias/default.asp $>$. Acesso em: Fevereiro de 2011.

TYRRELL, Sebastián (2000). The many dimensions of the software process. Crossroads. ACM, New York, p. 22-26. jun. 2000. 